FACTA UNIVERSITATIS (NIŠ)

Ser. Math. Inform. Vol. 36, No 1 (2021), 101-117

https://doi.org/10.22190/FUMI200322009T

Original Scientific Paper

\title{
ON THE EMBEDDING OF GROUPS AND DESIGNS IN A DIFFERENCE BLOCK DESIGN
}

\author{
Maryam Tale Masouleh ${ }^{1}$, Ali Iranmanesh ${ }^{1}$ and Henk Koppelaar ${ }^{2}$ \\ ${ }^{1}$ Faculty of Mathematical Sciences, Department of Mathematics, \\ Tarbiat Modares University, P. O. Box 14115-137, Tehran \\ 2 Faculty of Electrical Engineering, Mathematics and Computer Science, \\ Department of Computer Science, Delft University of Technology, Delft, Netherlands
}

\begin{abstract}
A difference BIBD is a balanced incomplete block design on a group, which is constructed by transferring a regular perfect difference system by a subgroup of its point set. There is an obvious bijection between these BIBDs and some copies of their point sets as two sets. In this paper, we investigate the algebraic structure of these block designs by defining a group-isomorphism between them and their point sets. It has been done by defining some relations between the independent-graphs of difference BIBDs and some Cayley graphs of their point sets. It has been shown that some Cayley graphs are embedded in the independent-graph of difference BIBDs as a spanning subgraphs. In order to find these relations, we find out a configuration ordering on these BIBDs, also we have also obtained some results about the classification of these BIBDs. This paper deals with difference BIBDs with even numbers of points.

Keywords. Balanced incomplete block design, sub-design, independent graph, Cayley graph, dihedral groups, configuration ordering.
\end{abstract}

\section{Introduction}

Let $G$ be a finite group of order $\nu(|G|=\nu)$ and $k, \lambda$ be two integers, where $k$ is less than $\nu$. A $t-(\nu, k, \lambda)$-balanced incomplete block design is an ordered pair $(G, \beta)$ such that $\beta$ is a family of $k$-subsets of $G$, named blocks, and every $t$ elements of $G$ do appear in exactly $\lambda$ blocks. For simplicity of notation, we write $(\nu, k, \lambda)$-BIBD (and some times $(\nu, k, \lambda)$-block design) instead of $2-(\nu, k, \lambda)$-balanced incomplete

Received March 22, 2020; accepted June 25, 2020.

Corresponding Author: Ali Iranmanesh, Faculty of Mathematical Sciences, Department of Mathematics, Tarbiat Modares University, P. O. Box 14115-137, Tehran, Iran | E-mail: iranmanesh@modarest.ac.ir

2010 Mathematics Subject Classification. Primary 05B05, 05C45 ; Secondary 05C51, $05 B 30$.

(c) 2021 by University of Niš, Serbia | Creative Commons License: CC BY-NC-ND 
block design. it will be called a trivial block design, when $k=\nu$. Suppose that $D$ is a $(\nu, k, \lambda)$-BIBD. A sub-design $D^{\prime}:\left(\nu, k, \lambda^{\prime}\right)$-BIBD of $D$ is such that every block of $D^{\prime}$ is a block of $D$ and this is denoted by $D^{\prime} \leq D$. Two block designs are isomorphic if there exists a bijection between the point sets such that blocks are mapped onto blocks. The embedding of a (family of) block designs into others are studied in $[7,11,12,14,22,23]$. Also there are some papers about the embedding of some block designs into some other mathematical objects like graphs [16], groups [1], surfaces or some applied mathematical concepts like (security of) coding, the mutually orthogonal [24], fast name retrieval in databases (named hashing) used for example in airports [5] or in social media [8]. A simple graph $\Gamma$ is an ordered pair $(V(\Gamma), E(\Gamma))$ consisting of a set $V(\Gamma)$ of vertices and a set $E(\Gamma)$, disjoint from $V(\Gamma)$, of edges, together with an incidence function $\rho \Gamma$ that associates with each edge of $\Gamma$ an unordered pair of vertices of $\Gamma$. A path $P_{n}$ is a simple graph with $n$ vertices whose vertices can be arranged in a linear sequence in such a way that two vertices are adjacent if they are consecutive in the sequence, and are non adjacent otherwise. A cycle $C_{n}$ is a $P_{n}$ such that the first and the last vertices are adjacent.

The aim of this paper is to bring together two areas in which a family of BIBDs have the same structure of groups. The first area is some of the block designs, whereas some graphs depend on them. The second area is the structure of groups as graphs.

Let $B_{1}, B_{2}, \ldots, B_{c}$ be $k$-subsets of $G$. For a finite group $G$, the difference of two elements of the group, say $x$ and $y$, is defined as $x y^{-1}$. Let $\Delta \beta$ denote the list of all possible differences between two blocks of $\beta ; \Delta \beta=\left\{x y^{-1} \mid x \in B, y \in B^{\prime}, B, B^{\prime} \in \beta\right\}$. Let $\mathcal{S}=\left\{B_{1}, B_{2}, \ldots, B_{c}\right\}$ be a subset of $\beta$. If every element of $G$ does appear exactly $\lambda$ times in $\Delta \mathcal{S}$, then $\mathcal{S}$ is called a $(\nu, k, \lambda)$-regular perfect difference system. This naming is in agreement with [21]. In the notation of [17], every element of this list is called an initial block and we will follow this notation. To shorten notation, we continue to write $(\nu, k, \lambda)$-d-system, for a $(\nu, k, \lambda)$-regular perfect difference system and only $\mathrm{d}$-system if there is no confusion. When a d-system has only one initial block, this block is well-known as a difference set. For a treatment of a more general case we refer the reader to $[3,19]$. The methods of constructing a d-system has been noted by many researchers. The best general reference and the classical work here is $[6,17]$.

Let $\theta$ and $y$ be two elements of the group $G$, the transference of $y$ by $\theta$ is equal to $\theta y$ and is denoted by $y^{\theta}$. The presentation $B=\left[\begin{array}{llll}y_{1} & y_{2} & \ldots & y_{k}\end{array}\right]$ of a block $B$ is used instead of $B=\left\{y_{1}, y_{2}, \ldots, y_{k}\right\}$ to avoid confusion with the set notation and to mention that a block is different from a usual subset of $G$. Assume that $A$ is a subset of $G$, maybe equals to $G$. A transference of block $B$ by a set $A$, is the set $B^{A}:=\left\{B^{\theta} \mid \theta \in A\right\}$, where $B^{\theta}=\left[\begin{array}{llll}y_{1}{ }^{\theta} & y_{2}{ }^{\theta} & \ldots & y_{k}{ }^{\theta}\end{array}\right]$. The transferring of a $(\nu, k, \lambda)$-difference system by its point set is a well known way to construct a BIBD, which is called a $(\nu, k, \lambda)$-difference block design or a $(\nu, k, \lambda)-d B I B D$ or briefly $d-B I B D$, when it will cause no confusion. Also we can do this transferring by a subgroup of $G$. It is easy to see and it is also well known that there is a bijection between a d-BIBD and some copies of $G$ as its point set (or some copies of one of its 
subgroups as a set, which is done the transferring of the d-system). The question, which arises here is: "Is this bijection a group-isomorphism?" In other words, does a d-BIBD have an algebraic structure as $G$ (or its subgroup) or some copies of it? Or this bijection is only a one-to-one function? Our view point sheds some new light on classification of d-BIBDs, finding the existence of some d-BIBDs and have a regular creatures, which are in math. In this paper, we investigate this problem and we can see that d-BIBDs have the same algebraic properties as their point set (Corollary 3.3). Also, as another result of proving the Lemma 3.1 and Lemma 3.2, we have the ordering of these designs, which can be applied to some groups. In fact, the d-BIBD inherited the algebraic structure of the point set. The corollary gains interest if we realize that it works for a bigger family of BIBDs. So we can see the extension of this method in Section 4. In the end, our theorems provide a natural and intrinsic characterization of these BIBDs (Theorem 4.1). These results can be applied to all d-BIBDs, as some corollaries, which are omitted in this paper. We can see a near view of these results about the automorphism of d-BIBDs in [19].

\section{Notation and Preliminaries}

Let $D:=(G, \beta)$ be a $(\nu, k, \lambda)$-BIBD, $b$ be the size of $\beta$ and $r$ be the number of blocks with one point of $G$ appearing in them. It is well known that

$$
\begin{gathered}
b=\frac{\vartheta(\vartheta-1) \lambda}{k(k-1)}, \\
r=\frac{(\vartheta-1) \lambda}{k-1} .
\end{gathered}
$$

Let $B=\left[\begin{array}{llll}y_{1} & y_{2} & \ldots & y_{k}\end{array}\right]$ be a block in $\beta$. As it was said in the introduction, a difference list of a difference system on $G$ is the list

$$
\Delta B:=\left\{Y_{i, j}:=y_{i} y_{j}{ }^{-1} \mid 1 \leq i, j \leq k\right\} .
$$

We want to use the transferring of difference system for building a BIBD on a non-Abelian group. According to this method, we can use this method for nonAbelian groups by fixing the direction of the group action from the left (or right) (For example, for a set $B=\{x, y, z\} \subseteq G$, for every $\theta \in G ; \theta B=\{\theta x, \theta y, \theta z\}$ ) as is done in [15]. This method will be denoted by LTDS (Left Transferring Difference System). The right and the left action have the same results up to isomorphism by a simple isomorphism function. We follow the LTDS on a non-Abelian group. From now on, all block designs are built by the LTDS unless it is mentioned.

The following theorem is useful about the structure of subgroups of $D_{2 n}$, where $D_{2 n}$ is dihedral group of order $2 n$, i.e., $D_{2 n}=\left\langle a, b \mid a^{n}=b^{2}=1 ; b a b=a^{-1}\right\rangle$.

Theorem 2.1. [9, Theorem 2.3] If $N$ is any proper normal subgroup of $D_{2 n}$, then $\frac{D_{2 n}}{N}$ is a dihedral group.

Theorem 2.2. [9, Theorem 3.1] Every subgroup of $D_{2 n}$ is cyclic or dihedral. A complete listing of the subgroups is as follows: 
1. $\left\langle a^{d}\right\rangle$, where $d \mid n$, with index $2 d$.

2. $\left\langle a^{d}, a^{i} b\right\rangle$, where $d \mid n$ and $0 \leq i \leq d-1$, with index $d$.

Every subgroup of $D_{2 n}$ occurs exactly once in this listing.

By Theorem 2.2 and [9], for every two dihedral groups, $D_{2 n}$ and $D_{2 m}$, either one is a subgroup of the other or both of them are subgroups of $D_{2 w}$, where $w=$ $\operatorname{lcm}(2 n, 2 m)$. Assume that $\Gamma$ and $\Upsilon$ are two graphs with vertex sets $V(\Gamma)$ and $V(\Upsilon)$, respectively. The adjoint of $\Gamma$ and $\Upsilon$, denoted by $\Gamma \vee \Upsilon$, is a graph with vertex set $V(\Gamma) \cup V(\Upsilon)$ and edge set $E(\Gamma) \cup E(\Upsilon)$. The Cartesian product of $\Gamma$ and $\Upsilon$ denoted by $(\Gamma \odot \Upsilon)$ is a graph such that its vertex set is the Cartesian product of $V(\Gamma)$ and $V(\Upsilon)$ and any two vertices $\left(u, u^{\prime}\right)$ and $\left(v, v^{\prime}\right)$ are adjacent in $\Gamma \odot \Upsilon$, if and only if either $u=v$ and $u^{\prime}$ is adjacent with $v^{\prime}$ in $\Upsilon$, or $u^{\prime}=v^{\prime}$ and $u$ is adjacent with $v$ in $\Gamma$. Let $G$ be a group and $S$ be a self-inverse and unit-free subset of it. The Cayley graph $\operatorname{Cay}(G, S)$ is a graph with vertex set $G$ such that two vertices $x$ and $y$ are connected by an edge if and only if $x y^{-1} \in S$. It's well known that $\operatorname{Cay}(G, S)$ is connected if and only if $S$ is a generator of $G$, see [13].

Theorem 2.3. [4] Let $C_{1}=\operatorname{Cay}\left(G, S_{1}\right)$ and $C_{2}=\operatorname{Cay}\left(H, S_{2}\right)$ be two Cayley graphs on groups $G$ and $H$, respectively. Then the Cartesian product $C_{1} \odot C_{2}$ is the Cayley graph $C=\operatorname{Cay}(G \times H, S)$, where $S=\left\{(x, 1),(1, y) \mid x \in S_{1} ; y \in S_{2}\right\}$ and $G \times H$ is the direct product of the groups $G$ and $H$.

Let $s$ be an integer and $d_{1}, d_{2}$ be two integers less than $s$. A Toeplitz graph $T_{s}\left\langle d_{1}, d_{2}\right\rangle$ is a graph with $\{1,2, \ldots, s\}$ as its vertex set and two integers $f$ and $f^{\prime}$ from $\{1,2, \ldots, s\}$ are adjacent if and only if $\left|f-f^{\prime}\right| \in\left\{d_{1}, d_{2}\right\}$.

Theorem 2.4. $\left[18\right.$, Theorem 2] $T_{n}\left\langle d_{1}, d_{2}\right\rangle$ decomposes into exactly gcd $\left(d_{1}, d_{2}\right)$ connected and isomorphic components.

Remark 2.1. Now let $G$ be a cyclic group of order $n$ as $\langle a\rangle$. Also $d_{1}$ and $d_{2}$ are two integers less than $n$. A Toeplitz graph $T_{G}\left\langle a^{d_{1}}, a^{d_{2}}\right\rangle$ is a graph with $G$ as its vertex set and two elements $f$ and $f^{\prime}$ of $G$ are adjacent if and only if $f f^{\prime-1} \in\left\{a^{d_{1}}, a^{d_{2}}\right\}$ or $f^{-1} f^{\prime} \in\left\{a^{d_{1}}, a^{d_{2}}\right\}$. As it is mentioned in [20], this graph is equal to the Cayley graph $\operatorname{Cay}\left(G,\left\{a^{d_{1}}, a^{d_{2}}\right\}\right)$. Note that there can be only one parameter for Toeplitz graphs.

\section{The even difference block designs}

One of the objectives in this section is to investigate difference block designs with point sets of even size. Furthermore, for doing this, we need some relations of these block designs, which can be found by algebraic properties of the point sets. In this section, we illustrate construction of a block design on dihedral groups by the LTDS method.

We are going to construct a block design $(2 n, k, \lambda)$-BIBD on the point set $D_{2 n}$, by the LTDS method. Let $\langle a\rangle=\left\{1=a^{0}, a, a^{2}, \ldots, a^{n-1}\right\}$ be a cyclic group of order $n$. 
Assume that there is a d-system with $c$ blocks $B_{1}, B_{2}, \ldots, B_{c}$ for a pair $\{k, \lambda\}$. Transfer these blocks by $\langle a\rangle$ from the left. There is a $(2 n, k, \lambda)$-BIBD by the LTDS. From now on, all block designs are supposed that are constructed by the LTDS on a d-system with $c$ blocks. The set $B_{i}^{H}$ will be called a family of blocks related to $B_{i}$, where $B_{i}$ is an initial block, $H$ is a subgroup of $G$ and $1 \leq i \leq c$.

Example 3.1. The $(10,3,2)$ - block design on $D_{10}$ with d-system:

$$
\begin{array}{ll}
B_{1} & =\left[\begin{array}{lll}
b & a b & a^{2} b
\end{array}\right], \quad B_{2}=\left[\begin{array}{lll}
a & a^{4} & b
\end{array}\right], \quad B_{3}=\left[\begin{array}{lll}
a^{2} & a^{3} & b
\end{array}\right], \\
B_{4} & =\left[\begin{array}{llll}
a & a^{4} & a^{2} b
\end{array}\right], \quad B_{5}=\left[\begin{array}{llll}
a^{2} & a^{3} & b
\end{array}\right], \quad B_{6}=\left[\begin{array}{lll}
1 & b & a^{2} b
\end{array}\right]
\end{array}
$$

is a set of blocks as follow:

$$
\begin{aligned}
& {\left[\begin{array}{lll}
b & a b & a^{2} b
\end{array}\right] \rightarrow\left[\begin{array}{lll}
a b & a^{2} b & a^{3} b
\end{array}\right] \quad\left[\begin{array}{lll}
a^{2} b & a^{3} b & a^{4} b
\end{array}\right] \quad\left[\begin{array}{lll}
a^{3} b & a^{4} b & b
\end{array}\right] \quad\left[\begin{array}{lll}
a^{4} b & b & a b
\end{array}\right]} \\
& {\left[\begin{array}{lll}
a & a^{4} & b
\end{array}\right] \quad \rightarrow \quad\left[\begin{array}{lll}
a^{2} & 1 & a b
\end{array}\right] \quad\left[\begin{array}{lll}
a^{3} & a & a^{2} b
\end{array}\right] \quad\left[\begin{array}{lll}
a^{4} & a^{2} & a^{3} b
\end{array}\right] \quad\left[\begin{array}{lll}
1 & a^{3} & a^{4} b
\end{array}\right]} \\
& {\left[\begin{array}{lll}
a^{2} & a^{3} & b
\end{array}\right] \rightarrow\left[\begin{array}{lll}
a^{3} & a^{4} & a b
\end{array}\right] \quad\left[\begin{array}{lll}
a^{4} & 1 & a^{2} b
\end{array}\right] \quad\left[\begin{array}{lll}
1 & a & a^{3} b
\end{array}\right] \quad\left[\begin{array}{lll}
a & a^{2} & a^{4} b
\end{array}\right]} \\
& {\left[\begin{array}{lll}
a & a^{4} & a^{2} b
\end{array}\right] \rightarrow\left[\begin{array}{lll}
a^{2} & 1 & a^{3} b
\end{array}\right] \quad\left[\begin{array}{lll}
a^{3} & a & a^{4} b
\end{array}\right] \quad\left[\begin{array}{lll}
a^{4} & a^{2} & b
\end{array}\right] \quad\left[\begin{array}{lll}
1 & a^{3} & a b
\end{array}\right]} \\
& {\left[\begin{array}{lll}
a^{2} & a^{3} & a^{2} b
\end{array}\right] \rightarrow\left[\begin{array}{lll}
a^{3} & a^{4} & a^{3} b
\end{array}\right] \quad\left[\begin{array}{lll}
a^{4} & 1 & a^{4} b
\end{array}\right] \quad\left[\begin{array}{lll}
1 & a & b
\end{array}\right] \quad\left[\begin{array}{lll}
a & a^{2} & a b
\end{array}\right]} \\
& {\left[\begin{array}{lll}
1 & b & a^{2} b
\end{array}\right] \quad \rightarrow \quad\left[\begin{array}{lll}
a & a b & a^{3} b
\end{array}\right] \quad\left[\begin{array}{lll}
a^{2} & a^{2} b & a^{4} b
\end{array}\right] \quad\left[\begin{array}{lll}
a^{3} & a^{3} b & b
\end{array}\right] \quad\left[\begin{array}{lll}
a^{4} & a^{4} b & a b
\end{array}\right] .}
\end{aligned}
$$

It's clear that if we multiply $b\left(b \in D_{2 n}\right)$ into families of blocks, then we have these families with the new names again. So by multiplying $b$ into initial blocks and transferring them, again we have a block design with the same parameters but different initial blocks. On the other hand, we know that the union of two block designs $D:\left(\nu, k, \lambda_{1}\right)$ and $D^{\prime}:\left(\nu, k, \lambda_{2}\right)$ is a block design $\bar{D}:\left(\nu, k, \lambda_{1}+\lambda_{2}\right)$. By the above notations, if the initial blocks of block design $D$ are transferred by $D_{2 n}$, which is the union of $\langle a\rangle$ and $\langle a\rangle b$, then there is a block design $\bar{D}$ with the same point set and the same block sizes but with a different parameter $\lambda$. By these, we can conclude the following remarks:

Remark 3.1. The difference block design $D_{1}:(2 n, k, \lambda)$ is isomorphic to a subdesign of d-BIBD $D_{2}:(2 n, k, 2 \lambda)$.

A difference block design on even points will be called the even block design.

Remark 3.2. Assume that $B=\left[\begin{array}{llll}y_{1} & y_{2} & \ldots & y_{k}\end{array}\right]$ and $B^{\prime}=\left[\begin{array}{llll}y_{1}^{\prime} & y_{2}^{\prime} & \ldots & y_{k}^{\prime}\end{array}\right]$ are two arbitrary blocks of an even block design. Suppose that they do not have any common elements. By a simple calculation, we can see that $B^{a}$ and $B^{\prime a}$ are disjoint, too.

\subsection{Even difference block design as a finite group}

In this section, we will provide some lemmas that we need for proving the main result. Also we can present the structure of even d-BIBDs by these theorems. The initial blocks are denoted by $B_{1}, B_{2}, \ldots, B_{c}$. In this case, for any initial block, the order of the points is arbitrary and fixed. Throughout this section, consider $G=D_{2 n}$ as the point set and $D=\left(D_{2 n}, \beta\right)$ is a $(\nu, k, \lambda)$-block design with $n \geq 5$. By Remark 3.1, apply the LTDS method with transferring of d-system by $D_{2 n}$, unless it is mentioned and also $\lambda$ is an even number. 
Definition 3.1. Assume that $A$ and $B$ are two sets such that $B \subseteq P(A)$, where $P(A)$ is the power set of $A$. The independent graph of $B$ is a graph with vertex set $B$ and two vertices are connected by an edge if and only if they are disjoint. The independent graph is denoted by $I G(A, B)$.

Base on design theory, the independent graph of a BIBD is a graph whose vertices are the blocks of this BIBD and two blocks are adjacent if and only if they are disjoint as two sets. The independent graph has its blocks as the vertices.

Lemma 3.1. Let $B_{i}$ be an initial block of design $D=\left(D_{2 n}, \beta\right)$, where $k<\frac{n}{3}$. Then the independent graph $I G\left(D_{2 n}, B_{i}{ }^{D_{2 n}}\right)$ is Hamiltonian.

Proof. It is sufficient to show that $I G\left(D_{2 n}, B_{i}{ }^{D_{2 n}}\right)$ has a spanning sub-graph, which is Hamiltonian. Let $d_{s}:=q\left(1 \leq s \leq \kappa=\left(\begin{array}{l}2 \\ k\end{array}\right)\right)$ if and only if there are $x$ and $y$ in $B_{i}$ with $x y^{-1}=a^{q} b^{\varepsilon}(\varepsilon=0,1)$. And define $\Delta B_{i}:=\left\{d_{1}, d_{2}, \ldots, d_{\kappa}\right\}$. Note that $\Delta \bar{B}_{i}$ is a set of integers modulo $n$. The proof will be divided into two cases:

Case 1. Assume that there is an integer such that is relatively prime to $n$ and belongs to $\{1,2, \ldots, n\} \backslash \Delta B_{i}$. Let $d$ be the minimum integer with this property. So by our assumption on $d$, it is clear that the set $B=\left\{B_{i, h}:=B_{i}{ }^{a^{h d}}\right\}_{h=1,2, \ldots, n}$ is a sequence of blocks such that $B_{i, h}$ and $B_{i, h+1}$ are independent for every $h$ and $h+1$ modulo $n$. This means that $I G\left(D_{2 n}, B\right)$ is a cycle $C_{n}$, by Remark 2.1. The lemma is proved for $B_{i}{ }^{H}$ with $H=\langle a\rangle$. Subtitute $\langle a\rangle$ and $B_{i}$ for $b\langle a\rangle$ and $B_{i}^{a}$, respectively. There will be another cycle, by applying some of the above methods. By Remark 3.2 , it is sufficient to find a block from $B_{i}{ }^{\langle a\rangle b}$, which is disjoint from $B_{i}$. If $B_{i}$ is a subset of $\langle a\rangle$ or $\langle a\rangle b$, then $B_{i}{ }^{b}$ is disjoint from $B_{i}$ and so we find a common edge. Assume that $B_{i}$ contains the elements $x \in\langle a\rangle$ and $y \in\langle a\rangle b$. Without loss of generality, suppose that $x=b y$ such that $x=a^{s} b^{\epsilon}$ and $y=a^{j} b^{\delta}$, where $s, j \in\{1,2, \ldots, n\}, \epsilon, \delta \in\{0,1\}$ and $\epsilon \neq \delta$. By multiplying $b$ by $y$ for obtaining $x=b y$, we have $a^{s+j-n} b^{\epsilon-\delta-1}=1$. It shows that $s+j \equiv 0(\bmod n)$. If there is another element of $B_{i}$, which is equal to $x$ after transferring by $b$, then it has to be equal to $y$, which is impossible. Now we can consider another case, $B_{i}{ }^{a b}$. Let $z$ be another element of $B_{i}$. By the above discussion, we know that $a x \neq a y$. If $a x=a b z$ $(a x=y), x=b z$ but $z \neq y$. If there are pairwise blocks with common element(s), due to the condition $k<\frac{n}{3}$, there is at least one block, which is disjoint from $B_{i}$, say $B^{\prime}$. There is $j \in\{1,2, \ldots, n\}$ such that $B^{\prime}=B_{i}{ }^{j} b$ (Fig.1). By Remark 3.2, the set $\left\{\left\{B_{i}{ }^{a^{s}}, B_{i}{ }^{s+j} b\right\} \mid 1 \leq s \leq n\right\}$ is a subset of edges of graph $I G\left(D_{2 n}, B_{i}{ }^{D_{2 n}}\right)$. Clearly, this graph is Hamiltonian and the proof is complete in this case.

Case 2. Assume that there is not any element of the set $\{1,2, \ldots, n\} \backslash \Delta \bar{B}_{i}$, where these integers are relatively prime to $n$. Let $d$ be the minimum element of the set $\{1,2, \ldots, n\} \backslash \Delta \bar{B}_{i}$ and $g:=\operatorname{gcd}(n, d)$. Remark 2.1 and our choosing integer $d$, show that the transferring of $B$ by $a^{d}$, forms a new graph. This graph includes $g$ isomorphic components, which every component is a spanning sub-graph of $I G\left(D_{2 n}, B_{i}{ }^{D_{2 n}}\right)$. Hence, the sequence $\left\{B_{i, h}:=B_{i}{ }^{{ }^{h d}} \mid h=1,2, \ldots, n\right\}$ forms $g$ isomorphic components by this transferring. Every component contains a spanning 


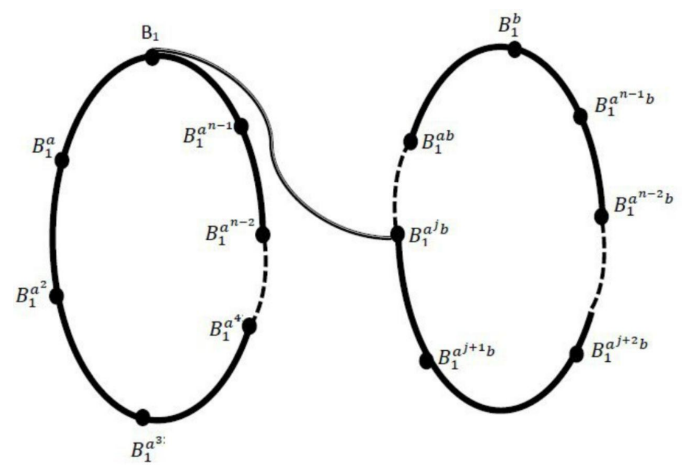

FIG. 3.1: The existence of an edge between two components of $I G\left(D_{2 n}, B_{i}^{D_{2 n}}\right)$.

cycle. So it is sufficient to find some edges between these components to obtain a Hamiltonian cycle. Let $d^{\prime}$ be an integer in $\{1,2, \ldots, n\} \backslash\left\{\Delta \bar{B}_{i} \cup\{d\}\right\}$, so there exists another element $d^{\prime}$ due to the condition $k<\frac{n}{3}$. Moreover, there is an edge $\left\{B_{i}, B_{i}{ }^{a^{d^{\prime}}}\right\}$ because $d^{\prime}$ is not in $\Delta \bar{B}$. On the other hand, every component is built by transferring the blocks by $a^{d}$ and for every vertex of $\operatorname{IG}\left(D_{2 n}, B_{i}{ }^{D_{2 n}}\right)$, this result can be applied by Remark 3.2, and hence the components are adjacent with more than one edge. Since every component contains a spanning cycle, so does $I G\left(D_{2 n}, B_{i}{ }^{D_{2 n}}\right)$.

As a consequence of the above Lemma, we have the following corollary.

Corollary 3.1. Let $B_{i}$ be an initial block of design $D=\left(D_{2 n}, \beta\right)$ and $d$ be as in the proof of Lemma 3.1 with, $k<\frac{n}{3}$ :

1. The graph $I G\left(D_{2 n}, B_{i}{ }^{D_{2 n}}\right)$ has a spanning sub-graph, which is isomorphic to $\operatorname{Cay}\left(D_{2 n},\left\{a^{d}, a^{-d}, b\right\}\right)$;

2. There is a bijection between $B_{i}{ }^{D_{2 n}}$ and $D_{2 n}$.

Lemma 3.2. For a difference block design $D$, if $k<\frac{n}{3}$, there is a cycle with $c$ vertices as a sub-graph of $I G\left(D_{2 n}, \beta\right)$ containing one and only one vertex from every $B_{i}{ }^{D_{2 n}}$, for $1 \leq i \leq c$.

Proof. Assume that there is a d-system of size $c$ equal to $\left\{B_{1}, B_{2}, \ldots, B_{c}\right\}$ with $c$ different difference lists $\Delta B_{1}, \Delta B_{2}, \ldots, \Delta B_{c}$, respectively. At first, we have to find an edge, which has $B_{1}$ as one of its vertices. Choose an element $B_{i}(2 \leq i \leq c)$ among all initial blocks. We continue the proof into two cases: either $B_{1} \cap B_{i}=\phi$ or $B_{1} \cap B_{i} \neq \phi$. In the first case, there is an edge between these blocks and we should go to the next step. But by the second case, we need to find a block from $B_{i}{ }^{D_{2 n}}$, which is disjoint from $B_{i}$. For doing this, we need defining the new sets. Put $\Delta B_{1, i}=\Delta B_{1} \cup \Delta B_{i}$ and $\Delta \bar{B}_{1, i}:=\left\{d \mid d \in\{1,2, \ldots, n\} ; a^{d} \in \Delta B_{1, i}\right.$ or $a^{i} b \in$ $\left.\Delta B_{1, i}\right\}$. Choose an integer belongs to $\{1,2, \ldots, n\} \backslash \Delta \bar{B}_{1, i}$ and denote it by $d_{1, i}$. 
Let $B_{i}^{\prime}:=\left(B_{i}\right)^{a^{d_{1, i}}}$. We can see that either $B_{1} \cap B_{2}=\phi$ or $B_{1} \cap B_{i}^{\prime} \neq \phi$. In the first case, we have the edge that we are looking for that. Suppose that there is at least one common element between $B_{1}$ and $B_{i}^{\prime}$. Then there are two cases:

1. There is no integer $d \in \Delta \bar{B}_{1}$ such that $d \mid d_{1, i}$ or $d_{1, i} \mid d$. So by transferring $B_{i}$ by $a^{d_{1, i}}$, we obtain the intended block.

2. There exists an integer $d \in \Delta \bar{B}_{1}$ such that $d \mid d_{1, i}$ (or $d_{1, i} \mid d$ ). In this case, by transferring $B_{i}$ by $a^{d_{1, i}}$ repeatedly, we will achieve some blocks, which are not disjoint from $B_{1}$. But $\left|B_{1}\right|=k$ and $k<\frac{n}{3}$, so we can choose another $d$ from $\Delta \bar{B}_{1, i}$. It allows us to look for this block (a block disjoint from $B_{1}$ ) between the vertices of other components in $I G\left(D_{2 n}, B_{i}{ }^{\langle a\rangle}\right)$. Note that by transferring $B_{i}$ by $a^{d_{1, i}}$ for $\operatorname{gcd}\left(d_{1, i}, n\right)$ times, if there is not a disjoint block from $B_{1}$, then we should choose another $d$ from $\Delta \bar{B}_{1, i}$. Now we find an edge between two blocks from two different families $B_{1}{ }^{D_{2 n}}$ and $B_{i}{ }^{D_{2 n}}$. Continue process for the gained block $\left(B_{i}^{\prime}\right)$ and the remaining initial blocks. Go on, until finding a path with $c$ vertices. Denote the last block of this path by $B_{j}^{\prime}\left(P_{c}: B_{1}, B_{i}^{\prime}, \ldots, B_{q}^{\prime}, B_{j}^{\prime}\right)$. The proof falls into two cases: either $B_{1} \cap B_{j}^{\prime}=\phi$ or $B_{1} \cap B_{j}^{\prime} \neq \phi$. In the first case, there is a cycle, which we were searching for. In other case, we need a restoration during the last step, when we are finding the last block $\left(B_{j}^{\prime}\right)$. We want to find a transference of block $B_{j}$ such that is disjoint from $B_{1}$ and $B_{q}^{\prime}$. Consider the value $d_{1, q, j}$ instead of $d_{q, j}$ to continue. This integer exists because of the condition $k<\frac{n}{3}$. By the above discussion, the transferring $B_{j}$ by the $\left(d_{1, q, j}\right)^{\text {th }}$ power of $a$, gives us a block, which is disjoint from these three blocks. The cycle is completed now and so is the proof (Figure 2).

Remark 3.2 guarantees that the transferring of the cycle, which is obtained in Lemma 3.2, forms $n$ isomorphic cycles in graph $I G\left(D_{2 n}, \beta\right)$. Let $S_{i}$ be the set $\left\{a^{d}, a^{-d}, b\right\}$, which is a subset of $D_{2 n}$ as it is mentioned in the proof of Lemma 3.1 , for every $B_{i}(1 \leq i \leq c)$. Also, we have seen that the graph $\operatorname{Cay}\left(D_{2 n}, S_{i}\right)$, for $1 \leq i \leq c$, is isomorphic to a spanning sub-graph of $I G\left(D_{2 n}, \beta\right)$ for a difference block design $\left(D_{2 n}, \beta\right)$, with $k<\frac{n}{3}$. The following corollary is an immediate consequence of the previous lemma.

Corollary 3.2. The Cartesian product of $\bigvee \operatorname{Cay}\left(D_{2 n}, S_{i}\right)$ and $C_{c}$, is isomorphic to a spanning sub-graph of $I G\left(D_{2 n}, \beta\right)$, with $k<\frac{n}{3}$.

To embed an even d-BIBD into a finite group we use difference systems and graph theory to find a relation between an even difference block design and a finite group. By definition, it is clear that the Cartesian product of two Hamiltonian graphs is a Hamiltonian graph. So by Corollary 3.2, we have a relation between groups and difference block designs. By the method of constructing the even d-BIBDs, the size of d-system, $c$ is equal to $\frac{b}{2 n}$. So by Corollary 3.1, Lemma 3.2 and Theorem 2.3, we have the following theorem.

Theorem 3.1. Assume that there is a difference block design $D:(2 n, k, \lambda)-B I B D$, with $k<\frac{n}{3}$ and an even number $\lambda$, such that its $d$-system is transferred by $D_{2 n}$. 
Let $c=\frac{(2 n-1) \lambda}{k(k-1)}$. Then there is a bijection between the group $D_{2 n} \times Z_{c}$ and the block design $D$.

The bijection of previous theorem is:

$$
\begin{aligned}
\Phi: \quad & \longrightarrow D_{2 n} \times Z_{c} \\
B & \longmapsto
\end{aligned}
$$

where $B$ is the transference of $B_{i}$ by $a^{j} b^{\epsilon}(j \in\{1,2, \ldots, n\})$. There is not any action defined between the elements of $\beta$. The action between any pair of blocks of $\beta$, say $B$ and $B^{\prime}$, will be defined as follows:

$$
B \odot B^{\prime}=\Phi\left(\Phi^{-1}(B) \Phi^{-1}\left(B^{\prime}\right)\right) .
$$

Corollary 3.3. Under the above assumption, $f:=\Phi^{-1}$ is an isomorphism from $D_{2 n} \times Z_{c}$ onto $\beta$.

We know that symmetric BIBDs are isomorphic to their point sets. Also, by definition, in these symmetric difference block designs $c=1$ and this isomorphism is comparable with the above corollary.

Now suppose that $\lambda$ is an odd number and equal to $2 L+1$, where $L$ is a positive integer. We saw in the proof of Lemma 3.1 that there are two cycles due to transference by $\langle a\rangle$ and $\langle a\rangle b$, which are connected by $n$ edges. For odd $\lambda$ 's, the transference can only be the group $\langle a\rangle(\langle a\rangle b)$, by the LTDS method. By Theorem 2.4, $I G\left(D_{2 n}, B_{i}{ }^{\langle a\rangle}\right)\left(I G\left(D_{2 n}, B_{i}{ }^{\langle a\rangle b}\right)\right)$ is a cycle or a union of isomorphic cycles. We now apply the above argument again, with $D_{2 n}$ or $d$ copies of $D_{g}$ replaced by $C_{2 n}$ or $d$ copies of $C_{g}$, respectively, where $d$ is as mentioned in proof of Lemma 3.1.

Corollary 3.4. Suppose that there is a difference block design $D:(2 n, k, \lambda)-B I B D$ with $k<\frac{n}{3}$, where $\lambda$ is an even or an odd integer such that its $d$-system is transferred by $\langle a\rangle$ of order $n$. Let $c=\frac{(2 n-1) \lambda}{k(k-1)}$. Then there is an isomorphism between the group $\langle a\rangle \times Z_{c}$ and the difference block design $D$.

Remark 3.3. Assume that there is a difference block design $D:(2 n, k, \lambda)$-BIBD with $k<\frac{n}{3}$ such that the d-system is transferred by $H$, where $H$ is a subgroup of $D_{2 n}$. Let $c=\frac{2 n(2 n-1) \lambda}{k(k-1)|H|}$. Then there is a bijection between group $H \times Z_{c}$ and the difference block design $D$.

Remark 3.4. Let $H$ be a subgroup of $D_{2 n}$ of order $n$, which is not cyclic and suppose that $4 \mid n$. By Theorem 2.1, $\frac{D_{2 n}}{\langle b\rangle} \cong\langle a\rangle$ and $\langle b\rangle \cong Z_{2}$. Hence doing the LTDS method with transferring by $H$, is a block design isomorphic to a difference block design on the point set $D_{2 n}$, which is achieved by transferring via $D_{n}$.

\subsection{Configurations and the ordering}

In this section, we will see that the Hamiltonian cycle, which is achieved in the proofs of Lemmas 3.1 and 3.2, gives us the ordering of even designs. 


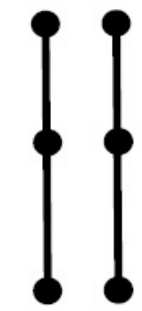

FIG. 3.2: Configuration $A_{1}$.

Definition 3.2. A $\nu$-configuration is a collection of $b$ lines (or subsets) having the property that every $t$-element subset is contained in at most $\lambda$ lines. And a $(\nu, l)$ configuration is a configuration of $p$ points on $l$ lines.

Definition 3.3. Let $D=(V, \beta)$ be a BIBD with $|\beta|=b$. Let $C$ be configuration on $l$ blocks. A Configuration ordering (or a C-ordering) for $D$ is a list of the blocks of $D, B_{0}, B_{1}, \ldots, B_{b-1}$, with the property that $B_{i}, B_{i+1}, \ldots, B_{i+l-1} \equiv C$ holds for all $0 \leq i \leq b-l$. If $B_{i}, B_{i+1}, \ldots, B_{I+l-1} \equiv C$ holds for all $0 \leq i \leq b-1$, with subscript addition performed modulo $b$, then the ordering is called $C-$ cyclic.

Let $A_{1}$ be a configuration as is mentioned in [10](part 4.1.1) and is shown in Figure 1 .

Theorem 3.2. [2] The existence of an $A_{1}$-cyclic ordering is equivalent to existence of a Hamiltonian cyclic in the independent graph of the block design.

It is known that the Cartesian product of two Hamiltonian graphs is Hamiltonian. So by these two lemmas (3.1 and 3.2) and Theorems 3.2, we have the following theorem:

Theorem 3.3. Every even block design with $k<\frac{\nu}{6}$, has the $A_{1}$-ordering.

Remark 3.5. According to the Corollary 3.3 , there is the $A_{1}$-ordering on the point sets of even block designs with $k<\frac{\nu}{6}$, by Theorem 3.3 .

\subsection{Even difference sub-designs and subgroups}

We have seen that some copies of dihedral groups are embedded into an even dBIBD with $k<\frac{n}{3}$. To find the relation between these BIBDs, we construct d-BIBDs by the LTDS method and find an isomorphism between these BIBDs and dihedral groups. Throughout this section, suppose that $k<\frac{n}{3}$ and $H$ is a subgroup of group G. 


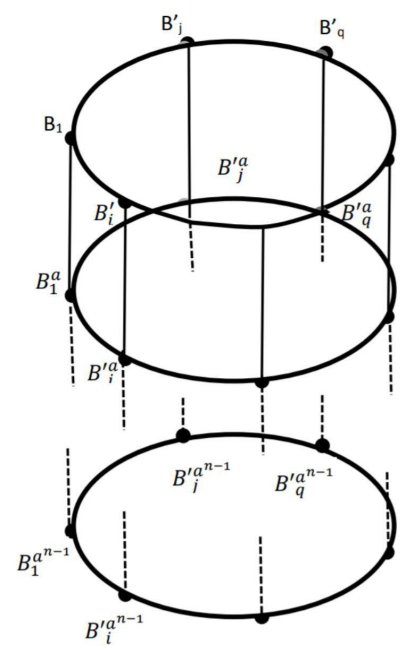

FIG. 3.3: The regular relations between cycles.

Definition 3.4. Let $G$ be a group and $H$ be a subgroup of $G$. A design-group $D H(G, k, \lambda)$ is an even difference block design $D$ with parameters $k$ and $\lambda$ with point set $G$ Also $\beta=\left\{B_{i}{ }^{H} \mid 1 \leq i \leq c\right\}$, where $H$ is a subgroup of $G$. A design-group $D K\left(G, k, \lambda^{\prime}\right)$ is a subdesign-group of $D H(G, k, \lambda)$ if $K$ is a subgroup of $H$ and even difference block design $\left(G, k, \lambda^{\prime}\right)^{K}$ is a sub-design of $(G, k, \lambda)^{H}$ as an even difference block design.

We can look at a design-group, both as a group and as a block design, simultaneously. Let $D_{1}:=D D_{2 n}\left(D_{2 n}, k, \lambda_{1}\right), D_{2}:=D H\left(D_{2 n}, k, \lambda_{2}\right)$ and $B$ be an arbitrary block of $D_{2}$ and $H=\langle a\rangle$. Note that the order of $a$ is $n$. Therefore, the blocks of $D_{2}$ are blocks of $D_{1}$ too. Because of the transference of its d-system by $\langle a\rangle$, it only has $c O(a)(=c n)$ blocks in $D_{1}$. For every $y_{j} \in B_{i},\left\{y_{j}{ }^{H}\right\}$ is a cosset of $H$ and is closed under the product of $H$ in itself. On the other hand, because of the same $c$ 's, for $D_{1}$ and $D_{2}$, the number of initial blocks is equal to $c$, but the number of $\theta$ 's is different. By calculating the number $c$ for both of them, if $k>3$, then according to equation (1), we obtain

$$
c=\frac{2 n(2 n-1) \lambda_{1}}{k(k-1) 2 n}, \quad c=\frac{2 n(2 n-1) \lambda_{2}}{k(k-1) n} .
$$

So we have $\frac{\lambda_{1}}{2}=\lambda_{2}$ and we can have Remark 3.1 as bellow by the new view of even block designs:

Proposition 3.1. Let $D D_{2 n}\left(D_{2 n}, k, \lambda\right)$ be an even design-group with even $\lambda$ and $H=\langle a\rangle$, then $D H\left(D_{2 n}, k, \frac{\lambda}{2}\right)$ is a subdesign-group of $D D_{2 n}\left(D_{2 n}, k, \lambda\right)$.

In fact, by changing the set of $H$ (the set, which the transferring of the LTDS method is done by that), we can control the possible values of $\lambda$. The last proposition shows 
us that for the point set $D_{2 n}$, if the d-system is transferred by the maximal subgroup $\langle a\rangle$, then we have a block design again. Now, what can we say about other subgroups of a point set?

Theorem 3.4. Let $D_{1}$ and $D_{2}$ be two difference block designs as $D H_{1}\left(D_{2 n}, k, \lambda_{1}\right)$ and $D H_{2}\left(D_{2 n}, k, \lambda_{2}\right)$, respectively with the same difference systems such that $\lambda_{2} \leq$ $\lambda_{1}$ and $k$ is odd.The even design-group $D_{1}$ can be embedded into the even designgroup $D_{2}$ if and only if $H_{1}$ can be embedded into $H_{2}$ as a subgroup.

Proof. By Theorem 2.2, it is sufficient to check two types of subgroups; $\left\langle a^{d}\right\rangle$ and $\left\langle a^{d}, a^{i} b\right\rangle(d \mid n$ and $i=0,1, \ldots, d-1)$. Let $D$ be a design-group $D D_{2 n}\left(D_{2 n}, k, \lambda\right)$ such that $\lambda_{1} \leq \lambda$. We will prove the theorem for difference designs $D$ and $D_{1}$,so it is true for every pair of designs. Let $B$ be an initial block of $D$. The proof naturally falls into the following two cases:

Case 1: Let $\theta \in\left\langle a^{d}\right\rangle$. This case is illustrated before in Proposition 3.1, for $d=1$. The block $B$ of $D_{1}$ is an element of $B^{\langle a\rangle}$. So $B^{\left\langle a^{d}\right\rangle}$ is a subset of $B^{D_{2 n}}$. Every difference block design has an independent graph. By the proof of Lemma 3.1 , to obtain a new difference block design, the deletion of blocks from $\beta$ has to follow a rule: Choose all $B_{i}^{a^{d}}$ and $B_{i}^{a^{d} b}$ such that $B_{i}$ is an initial block and there is a subgroup of $G$ of order $d$. So if $D_{1}$ is a sub-design of $D$, then it means that $H_{1}$ can be embedded into $D_{2 n}$ as a subgroup. Conversely, if $H_{1}<D_{2 n}$, then by the above facts about the choosing of blocks and existence of the independent graph, $D_{1}<D$ as a difference block design.

Case 2: Let $\theta \in\left\langle a^{d}, a^{i} b\right\rangle$. For every initial block $B$, the set $B^{\left\langle a^{d}\right\rangle}$ is a subset of $B^{\langle a\rangle}$, which was studied in Case 1 , and all remaining blocks in this set are in common with $B^{\langle a\rangle b}$. Again by Lemma $3.1, B^{\langle a\rangle b}$ and $B^{\langle a\rangle}$ are disjoint, for odd $k$ only. All blocks of $B^{\langle a\rangle b}$ are in common between two design-groups and others were studied in the previous case. The proof of the converse is similar to the above procedure.

\section{The second method and main result}

Sometimes there is no way to construct a BIBD on a point set with a special parameter. In these situations, there is a well known method: Assume that we want to have a BIBD on $(n+1)$ points. By this method, at first we construct a BIBD on group $G$ with $n$ elements and then we add an extra point, named $\infty$ such that $x \infty=\infty$, for every $x \in G$. So for having a difference BIBD, it is sufficient to add block(s) to the difference system such that the achieved system has the parameters, which we want. In this paper, this method will be called the LTDSE method ( the LTDS method with an Extra element). There are numbers of general examples of the LTDSE method in [17]. From now on, a d-BIBD which is based on the LTDS method will be called a type 1 BIBD and a d-BIBD, which is based on the LTDSE method will be called a type 2 BIBD. Note that the initial blocks of a type 2 BIBD are partitioned into two classes: a class of blocks including the extra variety $(\infty)$, which is of the size $r\left(\left\{B_{1}^{\prime}, B_{2}^{\prime}, \ldots, B_{q}^{\prime}\right\}\right)$ and the other class 
includes the remaining blocks $\left(\left\{B_{1}, B_{2}, \ldots, B_{p}\right\}\right)$. All facts about the transference of d-system $\left\{B_{1}, B_{2}, \ldots, B_{p}\right\}$ in Lemma 3.1 and Corollary 3.1, are applied for the first class of blocks of type 2 BIBDs.

Every type $1 \mathrm{BIBD}$ on even points is comparable to a type $1 \mathrm{BIBD}$ on $D_{2 n}$, and every type 2 BIBD on odd points is comparable to a type 2 BIBD on even points with an extra variety. For having this generalization on the point sets, at the end replace the integers by elements of dihedral group as below:

$$
2 i-1(1 \leq i \leq n) \longmapsto a^{i} \quad 2 i(1 \leq i \leq n) \longmapsto a^{i} b .
$$

Assume that $D$ is a d-BIBD. A natural question that arises here is "How can we find out whether 4 it is a type 1 BIBD or a type 2 BIBD?" From the previous sections, we know that replacing a subgroup of $G$, say $H$ in the LTDSE, leads us to a d-BIBD on $m n+1$ points with $\lambda$ less than the $\lambda$ of the d-BIBD, which is constructed by transferring its difference system by $G$. We have seen the relations between even d-BIBDs with point sets of similar size. But what can we say about two even dBIBDs with point sets of different sizes? What can we say about d-BIBDs in a general case?

Suppose that we can not see and detect the extra variety $\infty$ in blocks. Let $\Delta \beta$ be the list of all differences from the blocks of $\beta$ and $|\Delta \beta|=\psi \lambda+R$ (where $0 \leq R<\lambda)$. It is clear that $\frac{R}{\left(\begin{array}{c}k \\ 2\end{array}\right)}$ is the number of initial blocks, which are including the adjoined variety. First, we need some notations and observations:

Fact 1. By a review of the concept of the LTDS and the LTDSE methods, it is easy to see that $\psi$ is the size of the set, which transfers a d-system to obtain the d-BIBD and $\psi \mid b$. So we obtain the size of $H$, a subgroup of the point set that transfers d-system.

Fact 2. By doing the LTDS method and the LTDSE method, any element of $\Delta \beta$ is repeated symmetrically $\psi \lambda$ times for type $1 \mathrm{BIBD}$, and at least $(\psi \lambda+1)$ times for type 2 BIBD, By the definition of differences. Therefore the d-BIBD is of type 1 if $R=0$ and otherwise, it is a type 2 BIBD.

Fact 3. Assume that $B_{i}$ is a block of $\beta$ and $B_{i}{ }^{(H)}:=\left\{B \in \beta \mid \Delta B_{i}=\Delta B\right\}$. If $R=0$, then $\left|B_{i}{ }^{(H)}\right|=\psi$; otherwise, delete the blocks of $B_{i}{ }^{(H)}$, which includes the element $\nu$.

Fact 4. Assume that $d, d^{\prime} \in \Delta B_{i}$. To find the elements, which transfer $B_{i}$ to build $B_{i}{ }^{(H)}$, define $B\left(d, d^{\prime}\right):=\left\{(x, y, z) \mid, x y^{-1}=d, y z^{-1}=d^{\prime} ; d \neq d^{\prime}\right\}$. Then $H$ is equal to the set $\left\{x^{\prime} x^{-1}, y^{\prime} y^{-1}, z^{\prime} z^{-1} \mid \forall(x, y, z),\left(x^{\prime}, y^{\prime}, z^{\prime}\right) \in B_{i}\right\}$. From now on, all block designs are even difference block designs or a type 2 BIBD on an odd number of points.

According to Fact 4, we have a subgroup of the point set $H$, for every block design $D$, which is the transferrer for that d-BIBD; have a look at Theorem 2.2. 
Theorem 4.1. Let $D_{1}:\left(\nu_{1}, k, \lambda_{1}\right)$ and $D_{2}:\left(\nu_{2}, k, \lambda_{2}\right)$ be two difference block designs based on the LTDS or the LTDSE method. Let $c_{i}=\left\lfloor\frac{\left|\delta \beta_{i}\right|}{\lambda_{i}}\right\rfloor$ be the number of initial block(s) of the difference system of $D_{i}$ and $p_{i}, q_{i}$ are the numbers of initial blocks without $\infty$ and including $\infty$ in $D_{i}$, respectively as introduced in beginning of this section, for $i=1,2$.

1. Suppose that $\left|\Delta \beta_{1}\right|-\left\lfloor\frac{\left|\Delta \beta_{1}\right|}{\lambda_{1}}\right\rfloor=0$ or $\left|\Delta \beta_{2}\right|-\left\lfloor\frac{\left|\Delta \beta_{2}\right|}{\lambda_{2}}\right\rfloor=0$ and $\left\lfloor\frac{\left|\Delta \beta_{2}\right|}{\lambda_{2}}\right\rfloor$ is a multplied of $\left\lfloor\frac{\left|\Delta \beta_{1}\right|}{\lambda_{1}}\right\rfloor$. If $c_{p_{1}} \leq c_{p_{2}}$, then $D_{1} \leq D_{2}$, otherwise $D_{1} \leq\left\lceil\frac{c_{2}}{c_{1}}\right\rceil$ copies of $D_{2}$.

2. Suppose that $\left|\Delta \beta_{i}\right|-\left\lfloor\frac{\left|\Delta \beta_{i}\right|}{\lambda_{i}}\right\rfloor \neq 0$ and $\left\lfloor\frac{\left|\Delta \beta_{2}\right|}{\lambda_{2}}\right\rfloor$ is a multplied of $\left\lfloor\frac{\left|\Delta \beta_{1}\right|}{\lambda_{1}}\right\rfloor$. If $c_{p_{1}} \leq c_{p_{2}}$ and $c_{q_{1}} \leq c_{q_{2}}$, then $D_{1} \leq D_{2}$, otherwise $D_{1} \leq\left\lceil\frac{c_{2}}{c_{1}}\right\rceil$ copies of $D_{2}$.

Proof. Let $\left|\Delta \beta_{i}\right|=\psi_{i} \lambda_{i}+R_{i}$ such that $0 \leq R_{i}<\lambda_{i}$ and $c_{i}=\frac{b_{i}}{\psi_{i}}$, where $i \in\{1,2\}$. We follow the proof in two steps. We first compare an arbitrary family of each design according to their $H_{i}\left(B_{i}^{\left(H_{i}\right)}\right.$, for a block $B_{i}$ of a design) and then compare the number of these families, which is equal to $c_{i}$. The numbers $\nu$ and $\mu$ can be odd or even. As we have mentioned above, we illustrate difference block designs on even points for type 1 BIBDs and on odd points for type 2 BIBDs. Let $\nu=2 n+j$ and $\mu=2 m+j^{\prime}$, where $j, j^{\prime} \in\{0,1\}$. The numbers $2 n$ and $2 m$, where $m, n \in \mathcal{N}$, are called the even parts of the $\nu$ and $\mu$, respectively. Assume that $w=\operatorname{gcd}(2 n, 2 m)$ and $\nu \leq \mu$. So $D_{2 n}$ and $D_{2 m}$ are subgroups of $D_{w}$. If $\nu=\mu$ and the designs are type 1 BIBDs, then Theorem 3.4 lipids the relation between them. If $R_{i} \neq 0$ (for difference block design on $2 L+1$ points for a natural number $L$ ), then the initial blocks are of two types, as we have seen: the blocks including the adjoined variety $\beta_{q}:=\left\{B_{1}^{\prime}, \ldots, B_{q}^{\prime}\right\}$ and the blocks without the adjoined variety $\beta_{p}:=\left\{B_{1}, \ldots, B_{p}\right\}$. For that $q$ initial blocks, suppose that the new set of blocks is achieved by deleting the adjoined variety: $\beta_{q}^{\prime \prime}:=\left\{B_{1}^{\prime \prime}, \ldots, B_{q}^{\prime \prime}\right\}$ such that $B_{j}^{\prime \prime}=B_{j}^{\prime} \backslash\{\infty\}$, where $j \in$ $\{1, \ldots, q\}$. Note that maybe we do not see the adjoined variety as $\infty$, so there is an element out of the $D_{2 L}$ in blocks and also it is equal to $2 L+1$, where $2 L$ is the even part of the size of the point set. Also from now on, during the proof, $i$ belongs to $\{1,2\}$. According to the proof of Lemma 3.1, we are allowed to delete the blocks of every family by jumping $d$ steps, as it is mentioned there, unless the remaining blocks can not form a difference block design. This rule is the base of our work.

Step 1: Choose a block from each of the difference designs. Note, when we choose a block from $\beta_{i}$, it is an initial block (the representative of its family). In the next choice, we can choose another initial block by choosing every block, which doesn't have the same list of differences to the first block. As the first choice, assume that $B_{i}$ is an initial block of $D_{i}$. By Theorem 3.4, if $D_{1}$ and $D_{2}$ are type 1 BIBDs, then $B_{1} \subseteq B_{2}$ if and only if $H_{1} \leq H_{2}$. If $m, n$ are odd and $\psi_{1}, \psi_{2}$ have the same parity, then both of the $H_{i}$ 's are either cyclic groups or dihedral groups, by Theorem 2.2. Therefore, $B_{1} \subseteq B_{2}$ if and only if $\psi_{1} \leq \psi_{2}$. We need to know the relations between $H_{1}$ and $H_{2}$. There is an algorithm to know that $H_{i}$ is a cyclic subgroup or a dihedral subgroup of $D_{\omega}$. By the above Facts, $H_{i}$ is known. There are some cases: 
1. $H \leq\langle a\rangle$ such that $O(a)=w$;

2. $H \leq\langle a\rangle b$ such that $O(a)=w$ and $\operatorname{Ord}(b)=2$.

3. Otherwise, $H$ is a dihedral group $\left(|H|\right.$ is even) such that $\frac{\left|H_{i}\right|}{2} \mid \operatorname{Ord}(a)$.

Note that $\langle a\rangle b$ and $\langle a\rangle$ are isomorphic sets and one is a cosset of another in $D_{w}$.

1'. If both of the $H_{i}$ 's are of the form of case 1 (or 2), then $B_{1}^{(H)}<B_{2}^{(H)}$ if and only if $\psi_{1} \mid \psi_{2}$.

2'. If $H_{1}$ is of the form of case 1 or 2 and $H_{2}$ is of the form 3 , then $B_{1}^{(H)}<B_{2}^{(H)}$ if and only if $\psi_{1} \mid \frac{\psi_{2}}{2}$ (it yields $H_{1} \leq H_{2}$ ).

3'. If both of the $H_{1}$ and $H_{2}$ are of the form 3 , then $B_{1}^{(H)}<B_{2}^{(H)}$ if and only if $\psi_{1} \mid \psi_{2}$.

Now we are ready to illustrate the second step:

Step 2: Assume that $B_{1}{ }^{\left(H_{1}\right)}$ is a subgroup of $B_{2}{ }^{\left(H_{2}\right)}$ up to isomorphism. If $c_{1} \leq c_{2}$, then $D_{1} \leq D_{2}$. Otherwise, $D_{1}$ needs $\left\lceil\frac{c_{2}}{c_{1}}\right\rceil$ copies of $D_{2}$ to be embedded into, because $D_{1} \cong H_{1} \times Z_{c_{1}}$ and $D_{2} \cong H_{2} \times Z_{c_{2}}$. Also if $H_{1} \leq H_{2}$, then it has to have the case $Z_{c_{1}} \leq Z_{c_{2}}$, base on group theory (and $Z_{c_{1}} \subseteq Z_{c_{2}}$ ). But due to the condition $c_{2}<c_{1}$, it is impossible unless there are $\left\lceil\frac{c_{2}}{c_{1}}\right\rceil$ copies of $D_{2}$.

We study the case $R_{1}=R_{2}=0$ in steps 1 and 2 . If $R_{1}=0$ and $R_{2} \neq 0$, we apply the same manner as steps 1 and 2 on $\beta_{p_{1}}$ of $D_{1}$ and $\beta_{p_{2}}$ of $D_{2}$. If $R_{1}, R_{2} \neq 0$, then we apply the same arguments in steps 1 and 2 on $\beta_{p_{1}}$ of $D_{1}$ and $\beta_{P_{2}}$ of $D_{2}$ after that we do that on $\beta_{q_{1}}^{\prime \prime}$ of $D_{1}$ and $\beta_{q_{2}}^{\prime \prime}$ of $D_{2}$.

\section{Conclusion}

As the first step, we find the algebraic structure of difference block design on a dihedral group $\left(D_{2 n}\right.$ with arbitrary $\left.n\right)$ as its point set. We did that by finding the relation between its independent-graph and the Cayley graph of dihedral group with $S$, which is introduce during the proof of lemmas (Corollary 3.4). Due to our method to find this relation, we can prove that there exists a configuration ordering on these difference block designs (Theorem 3.3). Though the method of finding initial blocks can be from [17] or a lot of other references. At the end, we investigate these block designs, when they have an extra point. We present a method to recognize, when they are with an extra point or with the odd points. And finally, we can classify the big family of difference block designs, by presenting the Theorem 4.1. 


\section{Acknowledgement}

The authors would like to thank the anonymous referee for careful reading and the helpful comments improving this paper. This research of the first and the second authors was funded by a grant from INSF, Iran National Science Foundation [96004816].

\section{REFEREN C ES}

1. A. Caggegi and G. Falcone and M. Pavone: On the additivity of block designs. J. Algebraic Combin., 45(1) (2017), 271-294.

2. C. J. Colbourn and A. Rosa: Triple systems. OxfordMathematical Monographs, The Clarendon Press Oxford University Press: New York, USA, 1999.

3. C. J. Colbourn and J. H. Dinitz: Handbook of combinatorial designs, Second edition. Discrete Mathematics and its Applications (Boca Raton), Chapman \& Hall/CRC: Boca Raton, FL, 2007.

4. D. Cvetkovic and P.Rowlinson and S. Simic: An introduction to the theory of graph spectra. London Mathematical Society Student Texts, 75; Cambridge University Press: Cambridge, Britannia, 2010.

5. D. R. Stinson and R. Wei and L. Zhu: New constructions for perfect Hash families and related structures using combinatorial designs and codes. J. Combin. Des., 8(2000), No. 3, 189-200.

6. G. Ge and M. Ying and S. Xianwei: Perfect difference families, perfect difference matrices, and related combinatorial structures. J. Combin. Des., 18 (20180), No. 6, 65-78.

7. G. Lo Faro and A. Tripodi: Embeddings of $\lambda$ fold Kite systems, $\lambda \geq 2$. Australas.J. Combin., 36 (2006), 143-150.

8. J. Beel and B. Gipp: Google Scholar's Ranking Algorithm: An Introductory Overview. Proceedings of the 12th International Conference on Scientometrics and Informetrics. 1(2009), 230-241.

9. K. Conrad: Dihedral Groups. Retrieved from: http://www.math.uconn.edu/kconrad/blurbs/grouptheory/dihedral.pdf.

10. M. Dewar and B. Stevens: Ordering block designs, Gray code, Universal cycles and configuration orderings. CMS Books in Mathematics/Ouvrages de Mathematiques de la SMC; Springer: New York, USA, 2012.

11. M. Gionfriddo and S. Milici: Minimum embedding of a $K_{3}$-design into a balanced incomplete block design of index $\lambda \geq 2$ Ars Combin., 112 (2013), 279-291.

12. M. Meszka and A. Rosa: Embedding Steiner systems into Steiner systems $S(2 ; 4, \nu)$. Discrete Math., 274 (2004), No. 1-3 199-212.

13. PH. Zieschang: Cayley graphs of finite groups. J. Algebra, 118 (1988), No. 2, 447-454

14. P. J. Dukes and T. Feng and A. C. H. Ling: A finite embedding theorem for partial Steiner 3-designs. Finite Fields Appl., 33 (2015), 29-36. 
15. R. A. Bailey: Association Schemes Designed experiments Algebra and Combinatorics. Cambridge University Pres: New York, USA, 2004.

16. R. A Baily and P. J. Cameron: Using graphs to find the best block designs, Topics in structural graph theory. Encyclopedia Math. Appl., Cambridge Univ. Press, Cambridge, 147 (2013).

17. R. C. Bose: On the construction of balanced incomplete block designs. Ann. Eugenics, 9 (1939), 353-399.

18. R. Euler: Coloring planar Toeplitz graphs and the stable set polytope. 6th International Conference on Graph Theory, Discrete Math., 276 (2004), No. 1-3, 183-200.

19. R. Fuji-Hara and Y. Miao and S. Shinohara: Complete sets of disjoint difference families and their applications. Experimental design and related combinatorics. J. Statist. Plann. Inference, 106 (2002), No. 1-2, 87-103.

20. S. Bau: A generalization of the concept of Toeplitz graphs. Mong. Math. J., 15 (2011), 54-61.

21. S. Chisaki: difference systems of sets and their construction. Graduate School of Science and Technology; Tokyo University of Science: Tokyo, Japan, 2016.

22. S. Kucukcifci and G. Quattrocchi and B. R. Smith and E. S. Yzici: On regular embedding of H-designs into G-designs. Util. Math, 92 (2013), 97-127.

23. S. Milici: Maximum embedding of an $E_{2}(\nu \omega, 4,1)$ into an $H(\nu, 4, \lambda)$. Util. Math., 93 (2014), 383-391.

24. W. Ogataa and K. Kurosawa and D. R. Stinson and H. Saido: New combinatorial designs and their applications to authentication codes and secret sharing Schemes. Discrete Math., 279 (2004), No. 1-3, 383-405. 